\title{
Immunological Aspect in Inflammatory Bowel Disease
}

\author{
Darmadi Darmadi ${ }^{1 *(D)}$, Riska Habriel Ruslie ${ }^{2}$ \\ ${ }^{1}$ Department of Internal Medicine, Faculty of Medicine, Universitas Sumatera Utara, Medan, Indonesia; ${ }^{2}$ Department of Child \\ Health, Faculty of Medicine, Universitas Prima Indonesia, Medan, Indonesia
}

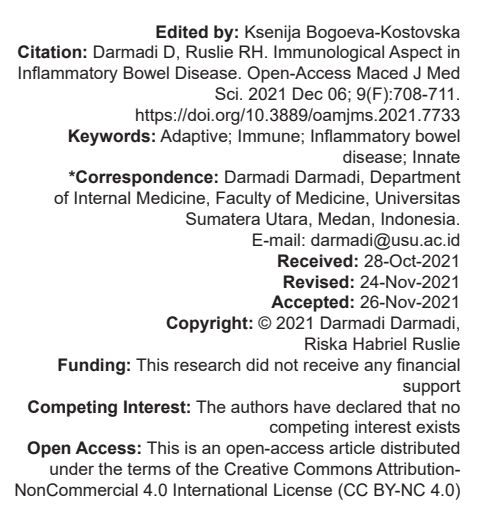

\begin{abstract}
Inflammatory bowel disease (IBD) is a chronic inflammation in the alimentary tract due to improper immune response toward external and internal antigens. The disease consists of two entities: Ulcerative colitis and Crohn's disease. The disease's prevalence is increasing worldwide due to westernization and industrialization. Europe still holds the highest prevalence of IBD in the world. There are two peaks of disease incidence. The first is in the third decade of life and the second is in the fourth decade. Slight male predominance is observed in IBD. Internal and external risk factors play an important role in the occurrence of IBD including genetic, smoking, reduced fiber intake, less or absent breastfeeding, sedentary occupation, pollution exposure, and medications. The disease carries heavy economic burden and hampers patient's quality of life. The immune concept of IBD was hypothesized in the 1950 s since the symptoms resolved with the administration of steroid. Innate and adaptive immune systems are involved in the pathogenesis of IBD. Antigen-presenting cells are found hyperactive, intestinal barrier is disrupted and autophagy activity is increased. Molecular mimicry occurs between foreign and self-antigen. The activity of $T$ helper (Th)1, Th2, and Th17 is amplified while regulatory T-cell's activity is suppressed. Pro-inflammatory cytokine production is elevated but anti-inflammatory cytokines are lowered. Finally, there is increased immunoglobulin $G$ level in intestinal mucosa and imbalance of gut microorganism. All the above immune disturbances lead to chronic inflammation in IBD.
\end{abstract}

\section{Introduction}

Inflammatory bowel disease (IBD) is a condition characterized by improper immune-mediated inflammatory response in the alimentary tract toward external and internal antigens. The inflammation occurs in chronic fashion [1], [2]. IBD consists of two subtypes which are ulcerative colitis (UC) and Crohn's disease (CD) [1], [3], [4]. Both have been identified since a long time ago. Wilks and Moxon were the first to identify UC in 1875 while CD was introduced by Burrill Crohn, Leon Ginzburg, and Gordon Oppenheimer later in 1932 [1], [3], [5].

CD affects all parts of gastrointestinal tract and manifests as fatigue, diarrhea with or without bleeding, abdominal pain, weight loss, and fever. UC itself affects colon and manifests as rectal bleeding, diarrhea, mucus discharge from rectum, tenesmus, and lower abdominal pain [6], [7]. The prevalence of IBD in Europe is the highest globally [3], [5]. Recently, IBD has spread to all regions in the world and becomes a global disease [3]. Fortunately, mortality rate from IBD is not significant. However, the disease carries heavy economic burden and hampers patients' quality of life. The economic burden is the result of hospitalization, surgery, ambulatory care, and medications. Annual direct health-care cost of IBD in Europe ranges from $€ 4.6$ to 5.6 billion [3]. In the USA, the economic burden from IBD exceeds 6 billion USD in 2004 [3], [4].

The concept of immunology in IBD began to rise in the 1950s, since the symptoms were ameliorated with the utilization of steroid [1]. Later, several immunomodulators are utilized for managing the disease such as thiopurine, infliximab, and methotrexate and show significant improvement [8], [9], [10]. Several risk factors are also associated with changing in host's immune system such as absence of breastfeeding during childhood [1], [3]. In this review, we will further discuss the immunological aspects of IBD.

\section{Epidemiology of IBD}

Nowadays, IBD is not an exclusive disease for Western world anymore. The incidence of IBD is reported to be increased globally [1], [4], [5]. The influence of lifestyle changing, namely, westernization and industrialization, in newly developed countries is suspected to be the cause of the increase [5], [7]. The incidence of $U C$ and $C D$ in Europe is reported to be 24.3 and $12.7 / 100,000$ persons per year, 
respectively, while in the USA is 12.2 and $10.7 / 100,000$ persons/year, respectively [4]. In Asia, the incidence of IBD ranges from 0.5 to $3.4 / 100,000$ population [11]. The prevalence of IBD is expected to be $660 / 100,000$ population in 2025 [3]. The peak incidence of IBD is in the third decade of life [1], [3], [12], while the second peak is in the fourth decade [12]. Gender predilection is comparable with slight male predominance, particularly in Asian countries [1], [12].

\section{Risk Factors of IBD}

Internal and external factors play role as risk factors of IBD. Internal risk factor includes genetic predisposition [6], [13]. Even though it cannot cause IBD independently, genetic is an important risk factor for the disease. Several genetic predispositions have been studied in association with IBD such as NOD2, IL23R, and ATG16L1 [3], [4], [6], [13], [14]. External factor includes broad spectrum of triggers. Hygiene theory is proposed to hold significant role in the occurrence of IBD. The incidence of IBD is increasing in line with increased hygiene level [3], [13], [15]. Increased hygiene level means less exposure to environmental antigens. This influences the balance between $T$ helper (Th)1, Th2, and regulatory $\mathrm{T}$ (Treg) cell. The imbalance affects immune response of host toward antigen exposure [12]. Impaired immune response tends to manifest later in advanced age [1], [3], [15]. Other risk factors associated with IBD are smoking, reduced fiber intake, less or absent breastfeeding, sedentary occupation, and pollution exposure [1], [3], [13]. Medications which can increase the incidence of IBD are oral contraceptive, hormonal replacement therapy, nonsteroidal anti-inflammatory drug, and antibiotic utilization [1].

\section{Immune System and IBD}

Normally, host's immune system maintains tolerance to commensal bacteria, self-antigens, and food antigens while protecting against harmful antigens by eliciting inflammation [2], [7]. Inappropriate immune response toward internal and external antigens is the hallmark of IBD. This situation leads to chronic inflammation [2], [12], [16], [17]. Almost all immune systems, including innate and adaptive, are affected in IBD. The antigens enter host's alimentary tract and being processed and presented by antigen-presenting cells (APCs) to $\mathrm{T}$ helper cells. In patients with IBD, APCs in the intestinal mucosa, such as macrophage and dendritic cell, are hyperactive. Their hyperactivity causes translocation of antigens from intestinal lumen which triggers inflammation [1], [2], [6], [17]. Activated APCs, such as dendritic cells and macrophages, signal the production of pro-inflammatory cytokines such as interleukin (IL)-1 $\beta$, IL-6, IL-18, and tumor necrosis factor (TNF) [2], [6], [13].

Intestinal barrier, another innate immune system, is also affected in IBD. Mucus layer and intestinal epithelium create a separated environment between gut lumen and systemic circulation. This is the first physical and chemical barrier encountered by antigens which enter digestive system. Interaction between enterocytes, goblet cells, neuroendocrine cells, Paneth cells, and $M$ cells is disrupted due to inflammation. Other environmental factors and infections also disturb the barrier's integrity. The disruption permits antigen translocation from intestinal lumen, triggers further inflammation, and creates a vicious cycle [2], [6], [7], [13], [14], [16], [17]. Besides increased permeability, disruption of barrier integrity also decreases the production of defensins, a bactericidal agent. Another innate immune mechanism, autophagy, is also involved in the inflammatory process in IBD. Autophagy is a mechanism to eliminate intracellular wastes including unfolded proteins. In subject with IBD, there is a tendency of increased intracellular wastes, therefore, autophagy activity is increased and creates a condition of chronic inflammation [7]. A study showed that mouse with intact innate immune system but disrupted adaptive immune system may not develop colitis. This shows the importance of innate immune system in the incidence of IBD [6].

Molecular mimicry between foreign and self-antigen is one of the triggers of chronic inflammation in IBD. The condition makes persistent inflammation even when the foreign antigen has been eliminated [18]. The APCs require human leukocyte antigen (HLA) on the surface of $T$ helper cells to be able to present the antigens. In subjects with IBD, the amount of HLA is increased, thus allowing better antigen presentation and processing [1], [2]. Interaction between APC and HLA on the surface of $T$ helper elicits broad immunological responses such as macrophage activation and TNF production. TNF production activates caspase and induces apoptosis. TNF also enhances other cytokines production and further orchestrates inflammation [18].

In CD, T-cell activity is amplified, mainly Th1 and Th17. Both cell lineages hold an important role in inflammation activity. They tend to elevate pro-inflammatory cytokine levels such as IL-17, interferon (IFN)- $\gamma$, and TNF- $\alpha$. On the other hand, Th2 activity is increased in UC. This leads to the production of IL-4, IL-5, and IL-13, activation of B cells, and natural killer cells [2], [6], [7], [13]. Newly identified effector T-cell, Th9, is also increased in UC patients. T helper 9 increases the production of IL-9, IL-4, and transforming growth factor (TGF)- $\beta$. IL-9 impairs gut barrier by inhibiting epithelial cell proliferation and disrupting tight 
junction [2]. Among all pro-inflammatory cytokines, TNF is considered as the primary cytokine in the immune response of IBD. TNF acts by amplifying inflammation and maintaining its chronicity. TNF also mediates crosstalk between innate and adaptive immune system in IBD [2], [6], [7]. Besides increasing IL production, lymphocyte $T$ cells also infiltrate gut mucosa and other extraintestinal organs, creating inflammation and various clinical manifestations [2]. In general, anti-inflammatory cytokines in subjects with IBD are downregulated, such as IL-2, IL-10, TGF- $\beta$, and TNF- $\beta$ [1], [2], [13]. Low IL-10 level prevents immunosuppressive effect on dendritic cells and macrophages. Meanwhile, low TGF- $\beta$ allows hyperactivity of macrophages and effector T-cells [2]. Regulatory T-cell, a subset of T-cells, has anti-inflammatory action. Regulatory T-cell's activity is lowered and associated with lower anti-inflammatory cytokines in IBD [2], [7]. The combination of imbalance between pro- and anti-inflammatory cytokine results in chronic inflammation in IBD [1], [2], [7]. The presence of cytokines prevents apoptosis of T-cells and maintains inflammation in host's body. On the other hand, cytokines induce necroptosis of mucosal lining cells. Cytokines also influence the extraintestinal symptoms of IBD. IL-6 is associated with liver manifestations while TNF is associated with arthritis and cachexia. The knowledge regarding the role of cytokine in IBD is utilized in therapeutic approach by administering anti-TNF [13], [14]. The result of anti-TNF administration is very favorable in inducing and maintaining remission of IBD [2].

The level of immunoglobulin $G(\lg G)$ and the rate of plasma cell infiltration in intestinal mucosa are increased in IBD patients [2]. The presence of IgG against gut microorganisms tends to trigger intestinal inflammation. This marks the loss of immune tolerance toward commensal microorganisms [2], [6]. In contrast, gut microorganisms have an important role in supporting immune system of gut. The growth of gut microorganism prevents the invasion of pathogenic microorganism to intestinal epithelial layer. Inflammation kills microorganisms in gut and decreases short-chain fatty acid production. Microorganism imbalance also disturbs Treg cell function and elevates inflammatory activity. In addition, gut epithelial function is hampered by microorganism imbalance and facilitates antigen translocation from gut lumen as the inflammation trigger [1], [6], [7], [13], [17]. The imbalance may be caused by several etiologies such as imprudent antibiotic utilization, viral infection, and fungal infection [6].

\section{Conclusion}

Both innate and adaptive immunities are involved in the pathogenesis of IBD. From innate immune system, there are hyperactivity of APCs, disturbance of intestinal barrier, increased autophagy activity, and molecular mimicry. Adaptive immune system is also impaired marked by amplified Th1, Th2, and Th17 activity, decreased Treg cell's activity, and elevated IgG in intestinal mucosa. Therefore, imbalance between pro- and anti-inflammatory cytokines occurs. In addition, there are loss of immune tolerance toward commensal microorganisms and imbalance of gut microorganism. The combination of above situations creates chronic inflammation and ends with IBD.

\section{References}

1. Malik TA. Inflammatory bowel disease historical perspective, epidemiology, and risk factors. Surg Clin North Am. 2015;95(6):1105-22. https://doi.org/10.1016/j.suc.2015.07.006 PMid:26596917

2. Ahluwalia B, Moraes L, Magnusson MK, Ohman L. Immunopathogenesis of inflammatory bowel disease and mechanisms of biological therapies. Scand J Gastroenterol. 2018;53(4):379-89. https://doi.org/10.1080/00365521.2018.144 7597

PMid:29523023

3. Kaplan GG. The global burden of IBD: From 2015 to 2025 Nat Rev Gastroenterol Hepatol. 2015;12(12):720-7. https://doi. org/10.1038/nrgastro.2015.150

PMid:26323879

4. Huang $\mathrm{H}$, Fang $\mathrm{M}$, Jostins L, Mirkov MU, Boucher G, Anderson CA, et al. Fine-mapping inflammatory bowel disease loci to single variant resolution. Nature. 2017;547(7662):173-8. https://doi.org/10.1038/nature22969

PMid:28658209

5. Ng SC, Shi HY, Hamidi N, Underwood FE, Tang W, Benchimol El, et al. Worldwide incidence and prevalence of inflammatory bowel disease in the $21^{\text {st }}$ century: A systematic review of population-based studies. Lancet. 2017;390(10114):2769-78. https://doi.org/10.1016/s0140-6736(17)32448-0 PMid:29050646

6. Wallace KL, Zheng L, Kanazawa Y, Shih DQ. Immunopathology of inflammatory bowel disease. World J Gastroenterol. 2014;20(1):6-21. https://doi.org/10.3748/wjg.v20.i1.6 PMid:24415853

7. Geremia A, Biancheri P, Allan P, Corazza GR, Di Sabatino A. Innate amd adaptive immunity in inflammatory bowel disease. Autoimmun Rev. 2014;13(1):3-10. https://doi.org/10.1016/j. autrev.2013.06.004

PMid:23774107

8. Guerra I, Algaba A, Jimenez L, Aller M, Garza D, Bonillo D, et al Incidence, clinical characteristics, and evolution of SARS-CoV-2 infection in patients with inflammatory bowel disease: A single-center study in Madrid, Spain. Inflamm Bowel Dis. 2020;27(1):izaa221. https://doi.org/10.1093\%2Fibd\%2Fizaa221

9. Macaluso FS, Orlando A. COVID-19 patients with inflammatory bowel disease: A systematic review of clinical data. Dig Liver Dis. 2020;52(11):1222-7. https://doi.org/10.1016\%2Fj. dld.2020.09.002 PMid:32928672

10. Verstockt B, Verstockt S, Rahiman SA, Ke B, Amauts K, Cleynen I, et al. Intestinal receptor of SARS-CoV-2 in inflamed 
IBD tissue seems downregulated by HNF4A in ileum and upregulated by interferon regulating factors in colon. J Crohns Colitis. 2021;15(3):485-98. https://doi.org/10.1093/ecco-jcc/ jjaa185

PMid:32915959

11. Amarapurkar AD, Amarapurkar DN, Rathi P, Sawant P, Patel N, Kamani $P$, et al. Risk factors for inflammatory bowel disease: A prospective multi-center study. Indian $\mathrm{J}$ Gastroenterol. 2018;37:189-95. https://doi.org/10.1007/s12664-018-0850-0 PMid:29987750

12. Mak WY, Zhao M, Ng SC, Burisch J. The epidemiology of inflammatory bowel disease: East meets west. J Gastroenterol Hepatol. 2020;35(3):380-9. https://doi.org/10.1111/jgh.14872 PMid:31596960

13. Neurath MF. Cytokines in inflammatory bowel disease. Nat Rev Immunol. 2014;14(5):329-42. https://doi.org/10.1038/nri3661 PMid:24751956

14. Bamias G, Arseneau KO, Cominelli F. Mouse models of inflammatory bowel disease for investigating mucosal immunity in the intestine. Curr Opin Gastroenterol. 2017;33:411-6. https://doi.org/10.1097/mog.0000000000000402

PMid:28901966

15. Gearry RB, Richardson AK, Frampton CM, DodgshunAJ, Barclay ML. Population-based cases control study of inflammatory bowel disease risk factors. J Gastroenterol Hepatol. 2010;25(2):325-33. https://doi.org/10.1111/j.1440-1746.2009.06140.x PMid:20074146

16. Zeng Z, Mukherjee A, Zhang H. From genetic to epigenetics, roles of epigenetics in inflammatory bowel disease. Front Genet. 2019;10:1017. https://doi.org/10.3389\%2Ffgene.2019.01017 PMid:31737035

17. Flynn S, Eisenstein S. Inflammatory bowel disease presentation and diagnosis. Surg Clin North Am. 2019;99:1051-62. https:// doi.org/10.1016/j.suc.2019.08.001

PMid:31676047

18. Greuter T, Vavricka SR. Extraintestinal manifestations in inflammatory bowel disease-epidemiology, genetics and pathogenesis. Expert Rev Gastroenterol Hepatol. 2019;13:307-17. https://doi.org/10.1080/17474124.2019.1574569

PMid:30791773 